International Journal of Current Advanced Research

ISSN: O: 2319-6475, ISSN: P: 2319 - 6505, Impact Factor: SJIF: 5.995

Available Online at www.journalijcar.org

Volume 6; Issue 3; March 2017; Page No. 2828-2901

DOI: http://dx.doi.org/10.24327/ijcar.2017.2901.0136

Research Article

\title{
THE KNOWLEDGE AND AWARENESS OF DENTAL PRACTITIONERS ON THE USES OF ANTI-MICROBIAL AGENTS IN MOUTHWASHES-A SURVEY
}

\author{
Rinieshah Nair R.Baskran., Dhanraj M and Preetham Prasad Nittala
}

Department of prosthodontics, Saveetha Dental College, Chennai

A R R I I C L E $\quad$ I N N F O

Article History:

Received $29^{\text {th }}$ December, 2016

Received in revised form $24^{\text {th }}$ January, 2017

Accepted $18^{\text {th }}$ February, 2017

Published online $28^{\text {th }}$ March, 2017

\section{Key words:}

Awareness, Anti-microbial mouthwash, Dental practitioners, Dental treatment.

\begin{abstract}
A B S T R A C T
Aim: This survey is conducted to have a wholesome understanding on the knowledge of dental practitioners on the various anti-microbial agents used in commercially available mouthwashes.

Materials and methods: This study is a survey on the knowledge of anti-microbial agents present in mouthwashes among 100 dental practitioners. It was carried out by using a selfadministered questionnaire with 9 multiple choice questions and 1 open ended question. The questionnaire was conducted by using survey planet which is an online survey site to make data collection faster and easier.

Results: This survey highlights that the aspect of oral hygiene in patients is accepted by all dentists as to be of utmost importance, and at the same time it brings out the lack of consensus among the treating specialist as to the ways to achieve it. Mouthwashes have only been prescribed very scarcely and $84 \%$ of the time is to treat dental carries. $86 \%$ of them said that chlorhexidine is the most plaque impeding antimicrobial agent present in mouthwash and $89 \%$ of them opted chlorhexidine as the staining agent in mouthwashes. Chlorhexidine on a regular basis could stain the mouth, and if the staining is severe, it could affect the teeth. This however can be treated by either undergoing professional prophylaxis or simply by brushing the teeth and tongue. Among commercially available mouthwashes, chlorhexidine was most commonly selected followed by hexetidine and others. The evidence available in the literature is relatively less given the magnitude of the issue. Through this survey we would like to stress upon the knowledge and awareness among dental practitioners regarding the use of antibiotic mouthwashes.

Conclusion: This survey proves to show that the knowledge of antibiotic mouthwashes among dental practitioners has no gaps that could affect patient safety.
\end{abstract}

Copyright $@ 2017$ Rinieshah Nair R.Baskran et al. This is an open access article distributed under the Creative Commons Attribution License, which permits unrestricted use, distribution, and reproduction in any medium, provided the original work is properly cited.

\section{INTRODUCTION}

Mouthwashes are chemical formulations that are mainly used as adjuncts to mechanical methods of plaque control and also in the treatment of various dental and medical conditions[1]. Dentists have a major role to play in the maintenance of good oral hygiene in the country[2-4]. Although several studies carried out in several countries have shown the role of mouthwashes in plaque control, there is an overall scarcity of information regarding the dentists' knowledge and attitude towards mouthwashes as agents of plaque control[5]. The main objectives of the study will be to establish the dentists' knowledge of mouthwashes in terms of their constituent ingredients, their short and long term side effects; their attitude towards the use of mouthwashes as plaque control agents and their use during treatment of various dental conditions.

*Corresponding author: Rinieshah Nair R.Baskran Department of prosthodontics, Saveetha Dental College, Chennai
A descriptive cross-sectional study will be conducted among dentists' in Chennai. A total of 100 dentists will be included in the study.

\section{MATERIALS AND METHODS}

This study is a survey on the knowledge of antimicrobial agents present in mouthwashes among 100 dental practitioners. The questionnaire was conducted via Survey Planet to allow a wider range of coverage. Survey Planet is an online survey site that makes data collection so much simpler. The collection of data was done by using a self-administered questionnaire with 9 multiple choice questions and one openended question.

\section{Questionnaire}

1. What is your age?

- 20-30

- 30-40

- 40-50 
- 50 and above

2. Are you specialised in any department in the field of dentistry? (open ended question)

3. Are you

- Male

- Female

4. How many times have you prescribed antibiotic mouthwashes to patients?

- $0-10$

- 10-20

- 20 and above

- Do not prescribe

5. For what reasondo you most commonly prescribe antibiotic mouthwashes?

- Dental carries

- Pulpitis

- Gingivitis

6. Which is the most plaque impeding antibiotic mouthwash?

- Chlorhexidine

- Hexetidine

- Triclosan

- Not sure

7. Which drug, when combined with zinc, has the highest microorganism inhibiting factor in the oral cavity?

- Chlorhexidine

- Hexetidine

- Triclosan

- Not sure

8. Which type of antibiotic mouthwash causes staining on the teeth?

- Chlorhexedine

- Hexetidine

- Triclosan

- Not sure

9. What are the polymers used to increase the retention of triclosan?

- Methoxylene and Maleic acid

- Methymethacrylate

- Methoxylene

- Maleic acid

- Not sure

10. How are phenolic agents beneficial as contents of antimicrobial mouthwash?

- Reduces plaque inhibition on teeth

- Supresses gingivitis

- Increases retention of plaque inhibitory agents

- All of the above

- None of the above

\section{RESULTS}

The results are displayed in a pie chart with properly calculated percentages.
D1 What s vour ace?

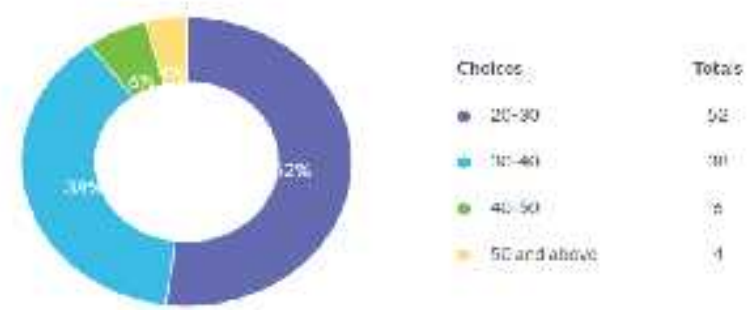

Q2 Areycu sperialised in any department in the feld of dentistry?
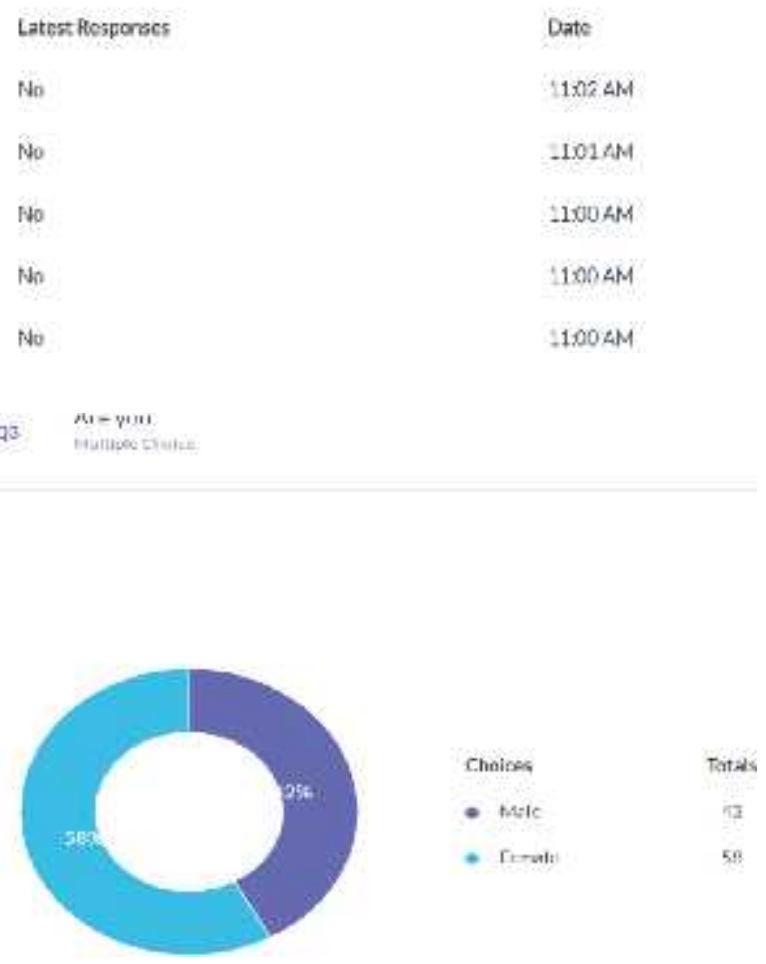
Q4 How many, times häwn yauprescriber ansiniatic mouthwaches to patients

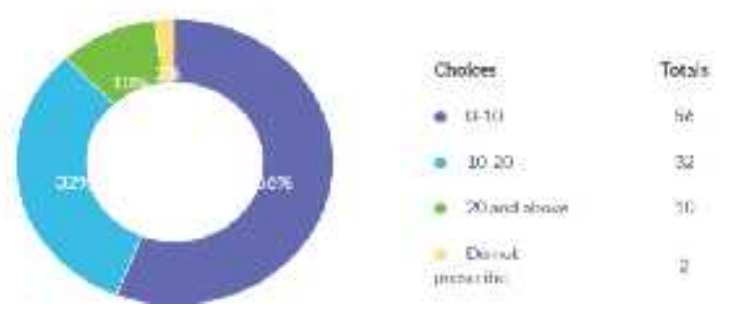




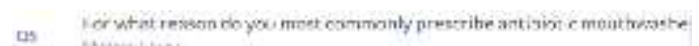
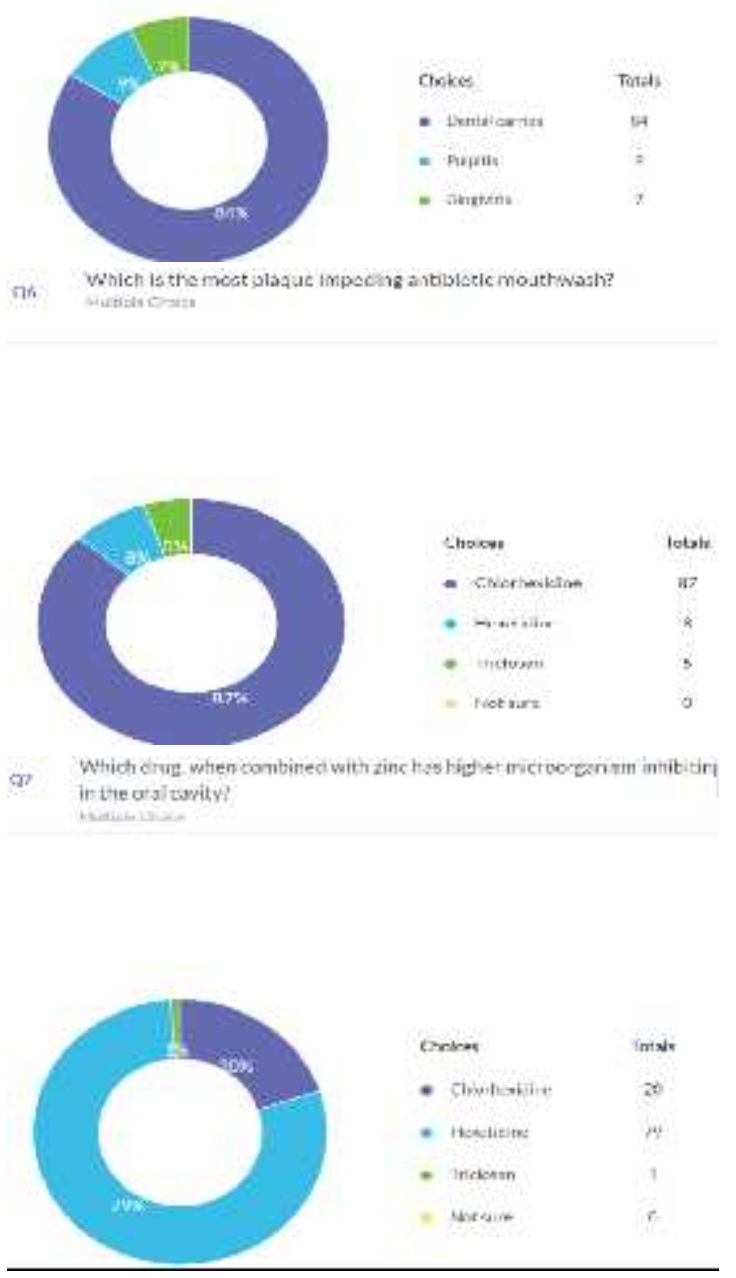

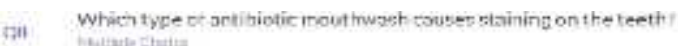
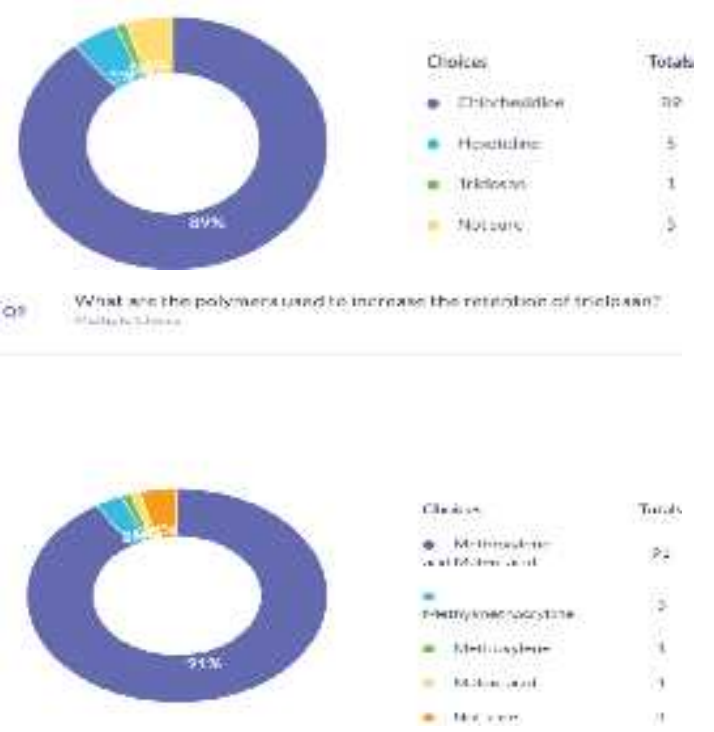

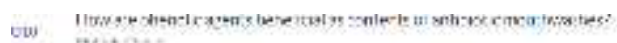

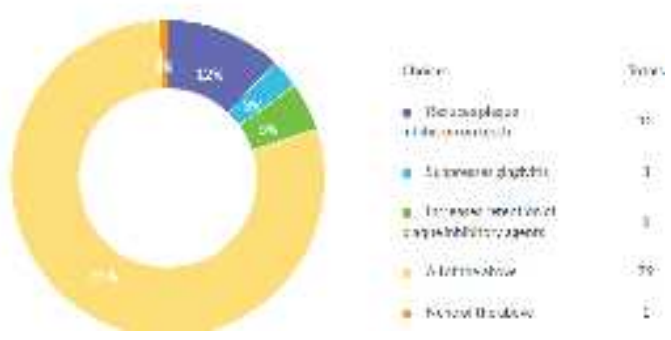

\section{DISCUSSION}

The questionnaire was distributed among 100 dental practitioners to assess their knowledge on antimicrobial agents present in mouthwashes. $52 \%$ of the dental practitioners are between 20-30 years of age and most of them are female dentists who are not specialised in any department in the field of dentistry. Mouthwashes have only been prescribed very scarcely and $84 \%$ of the time is to treat dental carries. $86 \%$ of them said that chlorhexidine is the most plaque impeding antimicrobial agent present in mouthwash and $89 \%$ of them opted chlorhexidine as the staining agent in mouthwashes. Chlorhexidine on a regular basis could stain the mouth, and if the staining is severe, it could affect the teeth. This however can be treated by either undergoing professional prophylaxis or simply by brushing the teeth and tongue. $79 \%$ of them said that hexetidine has higher microorganism inhibiting ability when combined with zinc. Methoxylene and Maleic acid are copolymers that have been opted by $91 \%$ of them as retentive agents for triclosan. $79 \%$ of them said that phenolic agents reduce plaque inhibition on teeth, suppresses gingivitis and increases retention of plaque inhibiting agents.

There exists non uniformity among prescription of mouthwash and the phase of therapy in which mouthwashes are prescribed. Among commercially available mouthwashes, chlorhexidine was most commonly selected followed by hexetidine and others.

According to various guidelines 8, 9, 10, 12 chlorhexidine is not to be prescribed for mucositis [6-8]. A pleasant tasting antimicrobial mouthwash formulation which maintains clarity for removal of dental plaque where no alcohol, sugar, artificial sweeteners are used making it suitable for safe use by alcoholics, diabetics, persons under medical treatment or taking medications which prelude the use of alcohol, hospitalized patients, prison inmates, minors and all other persons who can not or should not subject themselves to alcohol, sugar, or artificial sweeteners. The formulation consists of water, glycerin, sodium benzoate, cetylpyridinium chloride, citric acid, maltol, xylitol, a flavoring agent to give a pleasant though biting taste, and a coloring agent.

It is important to avoid all alcohol based mouthwashes in treating patients with oral mucositis.

As per various studies chlorhexetidine has shown improved ulcer healing with good analgesic, antimicrobial and antiinflammatory effect [8-11]. This survey highlights that the 
aspect of oral hygiene in patients is accepted by all dentists as to be of utmost importance, and at the same time it brings out the lack of consensus among the treating specialist as to the ways to achieve it. The evidence available in the literature is relatively less given the magnitude of the issue. Through this survey we would like to stress upon the knowledge and awareness among dental practitioners regarding the use of antibiotic mouthwashes.

\section{CONCLUSION}

This survey proves to show that the knowledge of antibiotic mouthwashes among dental practitioners has no gaps that could affect patient safety.

\section{References}

1. Addy, M., Chlorhexidine compared with other locally delivered antimicrobials. Journal of clinical periodontology, 1986. 13(10): p. 957-964.

2. Durack, D.T., Prevention of infective endocarditis. New England Journal of Medicine, 1995. 332(1): p. 3844.

3. Macpherson, L., et al., The role of primary healthcare professionals in oral cancer prevention and detection. British dental journal, 2003. 195(5): p. 277-281.

4. Slots, J. and T.J. Pallasch, Dentists' role in halting antimicrobial resistance. Journal of dental research, 1996. 75(6): p. 1338-1341.

5. Barkvoll, P., Actions and interactions of sodium lauryl sulfate and chlorhexidine in the oral cavity. 1991: Department of Pedodontics and Caries Prophylaxis, Dental Faculty, University of Oslo.
6. Garden, A.S. Mucositis: current management and investigations. in Seminars in radiation oncology. 2003. Elsevier.

7. Keefe, D.M., et al., Updated clinical practice guidelines for the prevention and treatment of mucositis. Cancer, 2007. 109(5): p. 820-831.

8. 8. Dodd, M.J., et al., Randomized clinical trial of the effectiveness of 3 commonly used mouthwashes to treat chemotherapy-induced mucositis. Oral Surgery, Oral Medicine, Oral Pathology, Oral Radiology, and Endodontology, 2000. 90(1): p. 39-47.

9. Miles, D.A., et al., Triamcinolone acetonide versus chlorhexidine for treatment of recurrent stomatitis. Oral surgery, oral medicine, oral pathology, 1993. 75(3): p. 397-402.

10. Matthews, R., et al., Clinical evaluation of benzydamine, chlorhexidine, and placebo mouthwashes in the management of recurrent aphthous stomatitis. Oral surgery, oral medicine, oral pathology, 1987. 63(2): p. 189-191.

11. Shaw, W., et al., Chlorhexidine and traumatic ulcers in orthodontic patients. The European Journal of Orthodontics, 1984. 6(1): p. 137-140.

\section{How to cite this article:}

Rinieshah Nair R.Baskran et al (2017) 'The knowledge and awareness of dental practitioners on the uses of Anti-microbial agents in mouthwashes-a survey', International Journal of Current Advanced Research, 06(03), pp. 2898-2901.

DOI: http://dx.doi.org/10.24327/ijcar.2017.2901.0136 